SHORT REPORT

\title{
RSV testing in bronchiolitis: which nasal sampling method is best?
}

\author{
P Macfarlane, J Denham, J Assous, C Hughes
}

Arch Dis Child 2005;90:634-635. doi: 10.1136/adc.2004.065144

Lower nasal swab and higher nasopharyngeal aspirate samples were compared for RSV immunofluorescence and pain score in infants hospitalised with acute bronchiolitis. The nasal swab procedure was significantly less painful but was negative in approximately one third of RSV positive cases.

aboratory confirmation of respiratory syncytial virus (RSV) is necessary for isolation or cohort nursing of -infants admitted with acute bronchiolitis. ${ }^{1}$ Sampling by nasopharyngeal aspirate (NPA) is commonly used but is invasive, painful, and requires equipment. The aim of this study was to test the hypothesis that simple nasal swab (NS) samples give similar RSV detection compared with NPA samples and that the NS sampling procedure is less painful than NPA in infants admitted with acute bronchiolitis.

\section{METHODS}

Infants hospitalised for management of suspected acute bronchiolitis were enrolled with written parental consent. Within 24 hours of admission each child had NPA performed through one nostril and NS from the opposite nostril. The sequence and laterality of the two sampling methods was allocated using a computer generated random number list. NPA specimens were collected by inserting an 8 FG catheter into one nostril to a depth equivalent to the distance between the infant's ear and nostril; vacuum suction was applied while gently retracting the catheter. If no sample reached the mucous trap, the catheter tip was cut off and placed in a universal container.

From the opposite nostril a nasal swab specimen was collected using a rayon tipped swab (Quadratech, UK) inserted about $2 \mathrm{~cm}$ into the nostril and rotated 2-3 times against the surface of the nasal cavity. The infant was swaddled for each procedure and settled between each sampling method.

Infant distress was measured during each procedure using a modified eight point facial, cry, and movement scoring scale. ${ }^{2}$ Parents were asked to judge which method caused least distress.

The paired samples were laboratory tested for RSV by immunofluorescence (Imagen RSV, UK).

A semi-quantitative estimate of respiratory epithelial cell numbers in each sample was made by microscopy.

The study protocol was approved by the local research ethics committee.

\section{RESULTS}

Paired samples were obtained from 88 children (mean age 6 months, range $1-20$ months). All samples contained sufficient epithelial cells for immunofluorescent testing. Table 1 shows the extent of agreement between the two sampling methods for positive and negative RSV results.
The prevalence of RSV in our study population by either sampling method was $38 \%(33 / 88)$. Both methods agreed for RSV positive in 24\% (21/88) and for RSV negative in 63\% (55/ 88 ), with 11 "false negative" NS and 1 positive NS when NPA was negative.

Using RSV positivity by either method as the gold standard (denominator) for presence of RSV; the sensitivity of NPA was $97 \%$ (32/33; CI $85-100 \%)$, whereas the sensitivity of NS was significantly lower at $67 \% \quad(22 / 33$; CI $\quad 51-83 \%$, $\mathrm{p}=0.0014)$.

Inherent from our gold standard definition, the positive predictive values for NS and NPA were both $100 \%$ (22/22 and $32 / 32$ ). The negative predictive value (the proportion of negative test results where RSV is absent on both tests) was $83 \%$ (55/66; CI 73-90\%) for NS, and for NPA was $98 \%$ (55/56; CI $91-100 \%)$.

The infant distress score $(\mathrm{n}=84)$ was significantly lower by NS (median 5), compared with NPA (median 6) $(\mathrm{p}<0.0001$, Wilcoxon rank sum test). This was consistent in all sub-scores and most marked for crying. Distress scores were not influenced by the order in which each of the sampling methods was applied (no sequence effect). Parents preferred NS (70\%) compared with NPA (15\%).

\section{DISCUSSION}

Our study showed that a simple nasal swab, while less painful for infants than NPA, failed to detect about one third of cases that were RSV positive by nasopharyngeal aspirate. Acute bronchiolitis is essentially a clinical diagnosis which is not dependent on viral testing, though the availability of a rapid RSV test does contributes towards clinical management by facilitating cohort nursing and infection control measures. ${ }^{1}$ The clinical availability of rapid viral tests for other respiratory viruses has also been shown to have a beneficial influence on clinical practice, antibiotic prescribing, and discharge planning. ${ }^{3}$

A previous study which involved older children showed that lower nasal swabs were significantly less painful than nasopharyngeal swabs; ${ }^{4}$ our study confirms a similar finding in infants, with respect to nasopharyngeal aspirates.

Why is NS less sensitive for RSV detection, compared with NPA? Immunofluorescence testing requires the presence of nasal epithelial cells on which to reliably perform the test. Importantly, in our study, the reduced sensitivity of NS samples for RSV cannot be explained by inadequate cell yield. All samples, both NS and NPA, contained sufficient nasal epithelial cells to yield a positive or negative result. This finding of reduced presence of RSV in the front of the nasal cavity compared with the nasopharynx is a consistent finding in two previous similar studies, both by culture ${ }^{5}$ and by immunofluorescence; ${ }^{6}$ this finding appears to be a consistent biological feature of RSV related to its preference for higher

Abbreviations: NPA, nasopharyngeal aspirate; NS, nasal swab; RSV, respiratory syncytial virus 


\begin{tabular}{|c|c|c|c|c|}
\hline \multicolumn{5}{|c|}{$\begin{array}{l}\text { Table } 1 \text { Extent of agreement between the two } \\
\text { sampling methods for positive and negative RSV } \\
\text { results }\end{array}$} \\
\hline \multicolumn{5}{|c|}{ NPA } \\
\hline & & + & - & \\
\hline \multirow[t]{3}{*}{ NS } & + & 21 & 1 & 22 \\
\hline & - & 11 & 55 & 66 \\
\hline & & 32 & 56 & 88 \\
\hline
\end{tabular}

ambient temperature, though not so for other common respiratory viruses. ${ }^{5}$

Nasal swab sampling has advantages over NPA; cost and equipment requirements are minimal, the method causes less pain and distress, but sensitivity is reduced. Nasal swab sampling may be more suitable for use outside of the hospital setting, where suction apparatus may not be available, or as a research tool in community studies to evaluate RSV epidemiology or prevention and treatment interventions.

Future research should evaluate RSV nucleic acid polymerase chain reaction testing, from different sites in the nose and pharynx, as a technique with the potential for increased sensitivity and which does not depend on retrieving infected epithelial cells. ${ }^{7}$

\section{ACKNOWLEDGEMENTS}

We are grateful to nursing staff who did the sampling and to Amanda Wilson who provided secretarial support to the project.
Authors' affiliations

P Macfarlane, J Assous, Department of Child Health, Rotherham

General Hospital, UK

J Denham, Department of Children's Nursing, Rotherham General

Hospital, UK

C Hughes, Department of Microbiology, Rotherham General Hospital, UK

Competing interests: none declared

Correspondence to: Dr P Macfarlane, Department of Child Health, Rotherham General Hospital, Moorgate Road, Rotherham S60 2UD, UK; peter.macfarlane@rothgen.nhs.uk

Accepted 2 February 2005

\section{REFERENCES}

1 Madge P, Paton JY, McColl JH, et al. Prospective controlled study of four infection-control procedures to prevent nosocomial infection with respiratory syncytial virus. Lancet 1992;340:1079-83.

2 Merkel SI, Joepel-Lewis T, Shayevitz JR, et al. The FLACC: a behavioural scale for scoring postoperative pain in young children. Pediatr Nurs 1997;23:293-7.

3 Byington CL, Castillo H, Gerber K, et al. The effect of rapid respiratory viral diagnostic testing on antibiotic use in a children's hospital. Arch Pediatr Adolesc Med 2002;156:1230-4.

4 Ipp $M$, Carson S, Petric $M$, et al. Rapid painless diagnosis of viral respiratory infection. Arch Dis Child 2002;86:372-3.

5 Heikkinen T, Marttila J, Salmi AA, et al. Nasal swab versus nasopharyngeal aspirate for isolation of respiratory viruses. J Clin Microbiol 2002;40:4337-9.

6 Stensballe LG, Trautner S, Kofoed PE, et al. Comparison of nasopharyngeal aspirate and nasal swab specimens for detection of respiratory syncytial virus in different settings in a developing country. Tropical Medicine and International Health 2002;7:317-21.

7 Jarti $\mathrm{T}$, Lehtinen $\mathrm{P}$, Vuorenen $\mathrm{T}$, et al. Respiratory picornavirus and respiratory syncytial virus as causative agents of acute expiratory wheezing in children. Emerging Infectious Diseases 2004;10:1095-101.

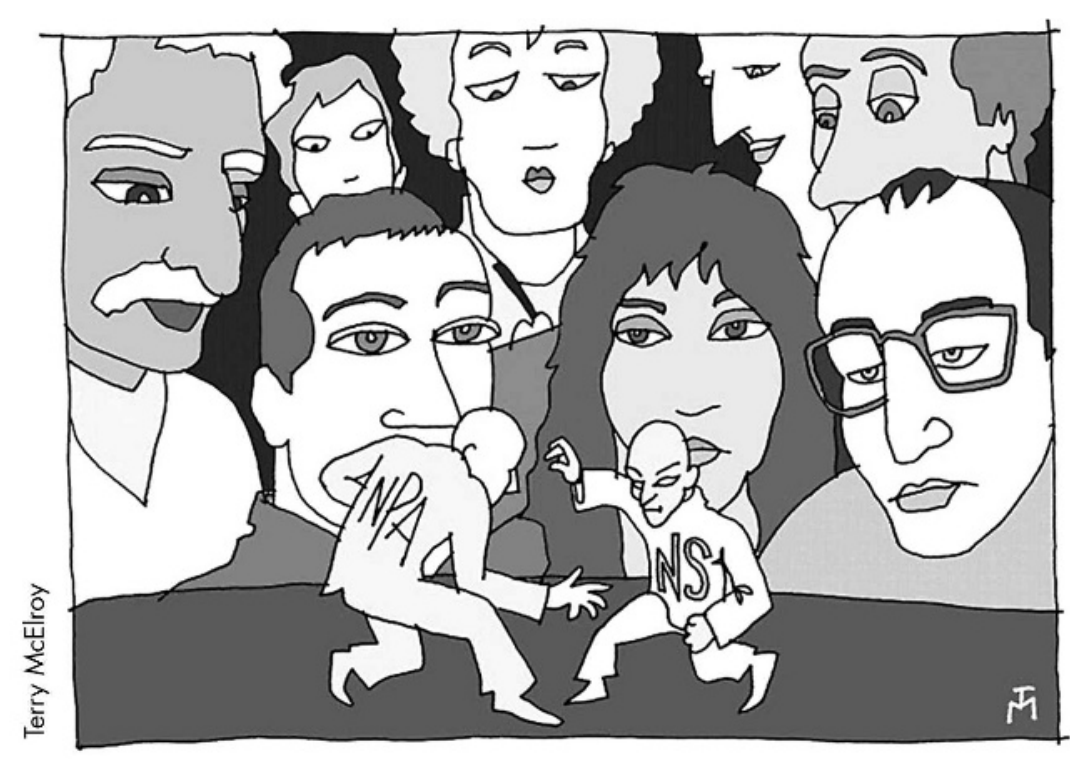

